\title{
NTEREACE
}

Sociologias, Porto Alegre, ano 10, no 19, jan./jun. 2008, p. 274-301

\section{La construcción de objetos socioeducativos con base epistemológica}

CARLOS E. MASSÉ NARVÁEZ*

\section{Motivos}

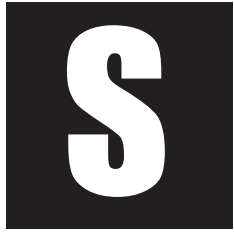

e parte de que es necesario abrir el debate sobre la debilidad presente en los proyectos de investigación educativa (IE). Esto se comprueba al identificar la falta de sustento teórico - epistemológico en la gran mayoría de los trabajos de investigación sobre el problema educativo. No pasamos por alto los esfuerzos realizados por diversos sectores de investigadores de la educación, que se presentan en los diversos foros nacionales que con ese fin se promueven; porque dichos trabajos están orientados a otros fines, de los que huelga decir son necesarios. Estos trabajos han aportado un gran número de investigaciones descriptivas muy útiles al estado del conocimiento sobre la problemática educativa en Latinoamérica y México. El mosaico que se recopila en estos eventos es no obstante el mejor indicador posible del estado de la (IE), como comúnmente se le concibe, y resulta ser tan diverso y multidimensional, que difícilmente se pudiera pretender abordar en su conjunto para los fines de éste trabajo. ${ }^{1}$

\footnotetext{
* Investigador de la Universidad Autónoma del Estado de México.E Mail: carmen311205@hotmail.com

1 Este texto es un avance inicial de una investigación más amplia que aspira a la creación de una propuesta de conocimiento para el ámbito socio educativo.
} 


\section{Problemática}

En Latinoamérica y México hay un fuerte ánimo e impulso para que la "investigación" ${ }^{2}$ se oriente hacia objetos de estudio en el ámbito de la educación, a los que sin mayor rigurosidad se les atribuye el mote de "campos". Sin embargo, son muy discutibles desde el punto de vista epistemológico un gran número de pretendidos campos existentes de estudios sobre problemas educativos y de otros de carácter emergentes que existen o surgen sin mayor rigurosidad científica y aunque algo aportan a constituir efectivamente nuevos campos, su constitución adolece del rigor señalado. Se entiende que su inicial taxonomía, en aras de abarcar la totalidad de la problemática en educación es necesaria; pero esto no debería dar pie a asignarles un objeto propio como campo o; incluso en ocasiones se cae en la confusión de que los campos pueden constituirse en una nueva disciplina.

Si observamos los avatares históricos del inicio del conocimiento científico y el de las ciencias sociales o, solamente alguna de ellas; como el caso de la historia de la Sociología como ciencia, o mejor aún el viejo debate que en torno al objeto de las ciencias humanas y, o sociales entre el naturalismo y el historicismo, y el actual entre naturalismo y ciencias sociales nos damos cuenta de que la cosa es mucho más sería de lo que podría pensarse sin profundidad. Solo contemporánemente podemos distinguir en Europa (en donde existe una gran tradición en las discusiones sobre el método), sobre la lógica de aprehensión - apropiación por ejemplo; o, sobre el asunto del relativismo en la ciencia, así como el retorno del sujeto, pasando por el paradigma de lo complejo, la especificidad frente al sistema, etcétera; sin dejar de lado el origen del conocimiento y sobre los fines del mismo, tan

2 Existe mucha confusión sobre el término, por ejemplo, a la preparación de una clase de un curso se le llama investigación; y, o a la hechura de una antología. A estas tareas de docencia se les llega a confundir con la investigación básica y, o aplicada y a la de frontera. Esta también se confunde con los "estados del arte", etc. 
solo recordemos la crítica de Popper al naturalismo y al historicismo marxista, pero también contra el inductivismo del Circulo de Viena (de Carnap y otros) y aún antes la de Marx frente a la filosofía especulativa; posteriormente los debates sobre la sociología alemana (en Tubinga, Alemania) entre Popper y los empírico analiticos (Albert) frente a la Escuela de Frankfurt (Adorno y Habermas), igualmente sobre un gran número de cuestiones epistemológicas nunca definidas de una vez y para siempre. Siempre en la arena de debate entre las ideologías y las metodologías. En la actualidad, un buen número de campos científicos han sido abordados por filósofos, sociólogos y naturalistas para la construcción de propuestas paradigmáticas como pueden ser: la Dialéctica Crítica (Bloch, Kosik, Zemelman, Covarrubias y quien suscribe), y el paradigma de lo complejo (Maturana, Morín, Luhmann).

Así las cosas, uno de los aspectos de esta gran problemática a la que queremos dedicar un poco de atención, es el asunto de cuestionar, por qué en el país (independientemente de que el problema seguro impregna otras latitudes latinoamericanas) la llamada "investigación educativa" (IE) adoptó acríticamente a la llamada teoría educativa y, o a la teoría pedagógica clásica. Cómo veremos más adelante, una pretendida perspectiva de base "experimental" no puede autoproclamarse poseedora del herramental de conocimiento para la investigación educativa y, o declarar que ese es el paradigma correcto.

Parte del atraso de nuestro país y en general de Latinoamérica, es la confusión reinante sobre lo que son: el conocimiento sin más, la investigación descriptiva, otra que se contenta con la prueba de teorías ya hechas y, la construcción de conocimiento científico con fundamentos sólidos y claros en el investigador. Esto es así, porque en la actualidad la auto-ctítica y la discusión escasean en el ámbito académico, dando paso a la implementación de investigaciones al interior de paradigmas cuyas bases epistemológicas son desconocidas para el propio investigador; realizando generalmente lo que khun llama "ciencia normal", con la agravante diferencia de que en el terreno de la investigación educativa en el país, la mayoría de la investigación que se hace no alcanza a constituirse en ciencia "normal", pues difícilmente 
podemos hablar en México de una comunidad científica en términos khunianos en las ciencias sociales por su gran complejidad y diversidad, por lo dicho líneas arriba; si hay por supuesto, comunidades donde se produce conocimiento específico pero corresponde a las ciencias llamadas duras. Por todo ello, se considera que buena parte de la investigación educativa (IE) en México se encuentra -en su mayoría - lejos de ser ciencia.

No existe novedad alguna en nuestra pretensión de señalar las carencias teóricas y, o el agotamiento explicativo de discursos teóricos que han sido hoy rebasados por la cambiante realidad. De hecho "la comunidad científica" global (así hay que decirlo ahora), ha "explicado" y vuelto a explicar innumerables problemas pedagógicos. Se reconoce sin embargo que la producción teórica no avanza al mismo ritmo sino mucho más lentamente. Esto es así por la complejidad que implica el proceso de teorización mismo. Sobre este aspecto abundaremos más adelante. De hecho "la comunidad" propuso alegremente -con ganas de salir de la crisis de los paradigmas-, el mito de la interdisciplinariedad, como pensando que varias disciplinas "aportan" más que una; al respecto, más adelante presentamos un esbozo de la problemática que implicaba solamente "montar" este pretendido macro - paradigma pero antes, a continuación presento un acercamiento al complejo mundo de la educación, para apoyar mi tesis de que las llamadas teoría educativas con base pedagógica no pueden dar cuenta de problemáticas que corresponden o corresponder deben a una multiplicidad de dimensiones de lo real, temporales, específico concretas, psicológicas, subjetivas etcétera.

\section{Esbozo de la multiplicidad de dimensiones que atraviesan al espectro educativo.}

En este apartado se alude a una mínima parte de la diversidad de dimensiones de lo real intrínsecas al objeto: transhistóricas, estructurales, prácticas disciplinarias, temporales, contextuales, etc. 
A continuación anotamos una serie de categorías y conceptos que, con diferentes connotaciones, son expuestos por diversos autores y aluden a una gran diversidad de problemáticas posibles tan sólo para que el lector se convenza de la complejidad a la que aludimos.

Obviamente, relacionado a un concepto amplio de educación se encuentra el concepto de finalidad en algunos de los autores que se han ocupado y los que se ocupan del asunto; o los fines de la educación. No obstante, en la mayoría de los casos la connotación de su significado se entiende como proveniente de sus diversos contextos histórico culturales. Lo que debe entenderse, como el conjunto de referentes empíricos y teóricos que circulan socializadamente en la época de los diversos autores; por ejemplo, con tintes liberales, conservadores, socialistas etc.

Al lado (no se quiere proponer un orden o jerarquía a los conceptos y categorías aquí enunciados) aparece el del sujeto (objeto de la educación), (su naturaleza); y se ha podido percibir también, una alusión preponderante a este sujeto, como al niño; por lo menos en autores del siglo pasado o incluso de la antigüedad. Esto aparece, por un lado, como algo muy natural; y por otro, al reflexionar esto con la realidad y la literatura actuales, se extraña que no se hable del adulto.

Si para algunos autores el fin de la educación es "sacar lo que está dentro del hombre"; supondría -en mi opinión—sacar "lo bueno" del individuo al educarlo. Por otra parte, la discusión sobre el sujeto, pasa necesariamente por la pregunta antropológica: ¿es el sujeto un ser bueno o malo?

$\mathrm{Y}$, en este planteamiento propongo que "lo malo" es la magnitud de la ignorancia. Podría decirse entonces, que se educa para reducir el nivel de la ignorancia; esto se vincula al concepto de necesidad (de la educación), concepto casi indiscutible -por demasiado obvio en nuestros días-; el cual se articula de diferente forma desde las distintas visiones de los autores; por ejemplo, con otro concepto de la mayor importancia, el de libertad. Para algunos la educación es necesaria porque libera al individuo de los mitos y prejuicios; que la falta de ésta (de la educación), permite que circulen 
pesadamente en la sociedad, pre - nociones y prejuicios de las diversas cosas, materiales e inmateriales (espirituales), que viajan como verdades para ciertas clases sociales impidiéndoles su liberación. Por poner un ejemplo, el concepto "par excellence" del discurso de la ilustración en el siglo XIX, sapere aude (atrévete a pensar por ti mismo), fue un concepto político frente al viejo orden de organización social (el feudal), que surge como parte del discurso de una clase social emergente (la burguesía) en ese contexto histórico cultural, como ariete para la liberación mental.

El mismo concepto de libertad de la educación es construido en el presente siglo por Paulo Freire, para luchar precisamente, contra de una corriente pedagógica a la que consideraba enajenante y alienadora. Perspectiva a la que consideraba nefasta y obstaculizante, para una libertad necesariamente requerida para el desarrollo cultural en general de los pueblos latinoamericanos.

Ahora bien, si vinculamos al concepto de cultura con el de creatividad; ambos en general, podrían aceptarse como indisolubles. Consideramos que una cultura o una época cultural, implica un desarrollo social (incluiría los niveles económico, político, cultural y psicosocial); es comparablemente mayor a otra en el desarrollo histórico, porque ha pasado por grandes momentos de creatividad. No entraré en la bizantina discusión de cuál fue primero, cual permitió que la otra se desarrollara. Lo interpretamos dialécticamente, como un proceso cuyas incidencias materiales y culturales (economía, política y cultura) articuladamente, impulsaron a esta última, la que se caracterizó por su mayor o menor creatividad. Ahora bien, creatividad puede ser también sinónimo de invención y de innovación en donde aquella incluye a estas últimas.

No podría estar ausente la problemática de los medios (los métodos) ${ }^{3}$ para adquirir la educación con los fines mencionados; sin embargo, en mi opinión el asunto es algo muy complejo y difuso. En la perspectiva en que

3 Es aquí tal vez en donde se encuentre la aportación de la (IE) "clásica"; no sin diferenciar entre métodos y técnicas y subrrayando que la (IE) se acota en este nivel y no a todos los objetos de investigación con ploblemáticas en educación. 
por el momento aquí se le concibe, no se exagera considerarlo además de un reto, como un campo de batalla entre las diferentes escuelas de pensamiento, autores y paradigmas; pues tanto la creación - asunción o adopción, con respecto a su uso, conlleva una concepción del mundo y tiene que ver, con la perspectiva de los fines. Es decir, el método está relacionado con la pregunta: ¿para qué y a quién? educar, con qué fin?

La problemática por abstracta que parezca, se articula empíricamente con el nivel institucional; con el sistema, los sistemas educativos. Con las universidades, pero también con los otros niveles escolares y con las distintas orientaciones, énfasis, etc. La educación de los niños en la escuela ortogénica; la aplicación psicoanalítica a la vida familiar; a los problemas sociales. Se pasa también por la reinterpretación del psicoanálisis (el que también es una hermenéutica), a las "medidas" en psicología. Así también, al estudio del individuo como objeto de la ciencia. Sin dejar, además, la preocupación latente del perfil del educador, profesional: ético, formativo, y actualmente "competitivo" —como el sistema quiere que sea—: (Durkheim).

Obviamente, también están presentes los asuntos de la Pedagogía: sus problemas didácticos fundamentales; la relación pedagógica, etc. La educación de adultos (sus tiempos culturales). El interés por educar, pero también por aprender. Educación con metas específicas, por ejemplo: para la paz; para la conservación del ambiente; para la "buena" sexualidad; para mejorar la ciudadanía, los derechos humanos, etcétera. Y en este punto detenernos para reflexionar sobre la función de la teoría (de la teoría pedagógica), pero también de la social y educativa; sin desatender la individualidad, el vínculo (sociología - psicología) habrá que dar lugar también a la visión genética; a los mecanismos del pensamiento y sus tiempos: a la psico - tempo - génesis.

En el mismo sentido, no se puede dejar de lado la relación entre dogmatismo y ética: entre ellos se arma el discurso político en general; el que vinculado a la retórica, puede armar el discurso demagógico; y, retornamos --por fuerza - al problema antropológico: de si la mayor capacidad del ser 
humano es hacer el mal (Nietzsche y Hobbes); o si es la de hacer el bien (Kant y Hegel). Y, tampoco, podemos dejar de articular a éste, con el discurso del vínculo cientificismo y progreso, cuya unión pseudoprogresista no resiste la pregunta: iprogreso para quién?

Tampoco son, todos éstos elementos, simples conceptos provenientes de discursos disciplinarios sin más; son elementos empíricos fundamentales del régimen monopólico ayer y hoy, bastiones del globalismo económico, pero también político y cultural. Su impacto en la educación es un tema actual de la mayor complejidad. Para su análisis, hay que acotar, sustanciar las expresiones, desdoblar los conceptos, estar a la casa de los fenómenos emergentes y su posible tendenciosa definición; ir de la Filosofía, a la Sociolgía y a la Psicología, y de ahí a la pedagogía; a la que no está de más una crítica epistemológica y ontológica.

Algo que igualmente no está fuera de este objeto (la educación), es estudiar el vínculo entre la escuela de la vida y la educación formal, distinguir aquella de la educación informal - "formal". Cómo vive el niño el paso del juego al trabajo "productivo". ¿Productivo para quién?: Preguntarse por la relación entre productividad y depredación ambiental y fáunica es obligado en un concepto amplio de educación. En política (concreta, educativa) ¿Quién, quienes, para qué, por que se delinean de una forma y no de otra. Cuáles requisitos hay que cumplir para ser tomador de decisiones de tales políticas; con qué argumentos, con qué bases se construyen; y cuál es la relación entre Conocimiento e Interés (Habermas). Más aún, preguntar con Foucault cuanta producción de saberes está siendo producida como base de la normalización y el control social? (escuela y poder).

En éste nivel, político también, cómo podemos articular lo anterior con la relación entre educación y democracia; y estos conceptos - problema, con el concepto, pero también con el proceso de globalización y exclusión, soberanía y nacionalismo en la educación. Individualismo o solidaridad. Se trata también de ver cómo inciden las reformas, pero también 
cuales son sus alcances de acuerdo a la estructura socialmente construida. Cómo ha incidido en todo esto la educación. ¿No se perfila ya, desde estos planteamientos como parte estructurante de los sistemas sociales?

Y finalmente (por el momento), ¿Cómo han incidido también las corrientes de las ciencias sociales volcadas al estudio de la educación, cómo lo abordan, qué sesgos le imprimen, cuáles son sus influencias en algunos autores, cuánto sesgo causa o puede causar, "enfocar" la educación desde esos discursos. ¿Se puede abordar aquella sin éstos; cuáles opciones existen desde la ciencia. ¿Se puede hablar de ciencia alternativa que lo permita?, y de ser así cual o cuales serían los paradigmas más sólidos para su abordaje?

Demostrar que no sólo se puede sino que debe hacerse ya, es uno de los principales objetivos de ésta investigación que apenas empieza; la cual debía haberse hecho, mucho antes de que, arbitrariamente y sin mayor fundamento, algunos de estos discursos descriptivos y empíricos sin más se constituyeran en supuestos campos con sus discursos disciplinarios denominados científicos (con teoría, objeto y método propios); y también incursionar en éstos para mostrar su desnudez sistemática.

Pero enunciar esta multiplicidad de dimensiones del objeto, tiene por finalidad preguntar al lector ¿Toda esta multidiversidad problemática, analíticamente (Descartes), no podría encuadrarse a nivel general para su estudio, en no más de cuatro niveles de realidad (teóricos si se quiere): el nivel político, el nivel económico, el nivel cultural y el psicosocial? Y, a partir de ahí ir pensando en la multiplicidad también de disciplinas cuyos hábeas teóricos tendrían algo que decir sobre el espectro educativo; así como pensar también en la gran complejidad que significaría su articulación sistemática en la construcción de conocimiento científico posible?

Ahora bien, ante esta gran diversidad de dimensiones y conexiones entre fenómenos que atraviesan tan variada problemática, existe una disciplina: la Sociología; la la que siendo subdividida en múltiples recortes va dando cuenta de los muchos campos de la educación; casi siempre a partir 
de cuerpos teóricos ya construidos y con ello va aportando conocimiento sobre algunos campos. No obstante, existe poca teorización sobre los mismos; aún cuando se actúa metodológicamente dentro del paradigma general llamado "método hipotético deductivo", el cual se conforma con "explicar" con base en pruebas de hipótesis la realidad educativa estudiada. Como se verá en nuestra propuesta, esta es una limitación que fue ya señalada desde los años treinta por Karl Popper y que sin embargo continúa vigente en los investigadores de todas las latitudes.

\section{Alcance y límites de la sociología de la educación}

El planteamiento implícito en este enunciado intenta ir fraguando el camino que abra paso a la sustentación de una perspectiva epistemológica para el estudio de la educación. Para ello se exponen algunos elementos de diversas perspectivas de la sociología de la educación y otros enfoques sobre el problema educativo con puntos de apoyo para la identificación de los fundamentos que sustentan a dichos discursos, con el fin de construir los más idóneos para a investigación educativa como investigación científica. Más allá de los límites disciplinarios teóricos de esta o aquélla disciplina, incluyendo a la misma sociología de la educación.

Otra cuestión es, la necesidad de aclarar por qué se incluye en éste trabajo a la sociología de la educación y no a algún otro paradigma que estudia a la educación. El argumento es que la sociología de la educación, incluye una visión explicativa amplia de la misma, es decir, racionaliza y. o, la puede cuantificar desde esa visión teórica global que es la sociedad. Implica por ello, una multidimensional visión de la misma: en su relación con el Estado, con la política, con la economía, con la cultura (de la que forma parte), etcétera. Sin embargo, el agotamiento de los alcances explicativos teóricos caducan por cuestión de tiempo. De ahí la necesidad de insistir en la búsqueda de una propuesta más firme que la sola teoría. Es 
decir, una propuesta de base epistemológica. Un argumento fuerte es la caída en cuenta de los dialéctico - críticos y de los posmodernos (Morín) de que la fragmentación del conocimiento por disciplinas condujo al estancamiento del conocimiento y, o al conocimiento limitado y sesgado del positivismo). Más adelante hablaremos del origen de dicha fragmentación. La sociología de la educación (clásica)

Existen diversos enfoques de la Sociología Clásica que me atrevería a dividir en tres grandes corrientes generales, de las cuales se desprenden otras muchas más específicas, según existan propuestas de conocimiento sociológico sobre la educación. Entre las primeras encontraríamos a los representantes ya considerados Clásicos de la sociología: Dhurkheim, Weber y Marx.

El primero si escribió específicamente al nivel ontológico sobre la educación, (ésta ha de ser como la sociedad específica quiere que sea) y metodológicamente hablando, dejó un legado genérico para el estudio de cualquier hecho social, objeto de la sociología

El segundo autor se orientó más a los estudios de economía, burocracia y al de las religiones en general. Se dijo arriba que metodológicamente hablando, dejó un legado breve pero claro acerca del abordaje de los problemas sociales como objeto de la sociología y de cómo tipologizarla. En él cabría -si se me permite_obviar que, para éste autor, la educación sería un objeto implícito en su obra; ya que a ésta el mismo le ha catalogado como a "las ciencias de la cultura". De hecho su concepto de profesión implica una determinada educación (religiosa o laica) que distingue culturalmente a algunas sociedades que las practican.

En el caso de Marx no se encuentran escritos específicos sobre el asunto de la educación; pero a través de sus conceptos de: ideología, estado y superestructura, algunos marxistas posteriores, fueron construyendo discursos explicativos sobre el papel de la educación en la sociedad capitalista. Por ejemplo, Luis Althusser la concibió como un aparato ideológico de control del Estado Burgués, a la manera como Marx concebía a éste. 
Resumiendo, podría decirse que la sociología "clásica" ha contribuido de alguna manera en la construcción de discursos teórico - explicativos sobre las causas o factores que inciden en la problemática educativa, tanto cuando a ésta se le fundamenta como en proceso de evolución, tanto cuando se argumenta en su contra (como obstáculo para la libertad). A Durkheim se le va a atribuir su influencia en el funcionalismo norteamericano y a Weber las raíces del estructural - funcionalismo también norteamericano y sajón en general; y a Marx y sus epígonos, la crítica de la cultura (incluye a la educación), y conlleva el énfasis en el elemento liberación.

Aunque fue Durkheim el primero en abordar la educación como rama particular de la sociología, le antecedieron Saint Simon y Comte; quienes al observar la transición de la sociedad del Antiguo régimen a la sociedad industrial a lo largo del siglo XIX, con la consiguiente preocupación por el desorden moral derivado de la creciente diferenciación - estratificación social, se concede a la educación la noble tarea de garantizar -a través de ella-, la comunión moral de la sociedad y la cohesión partiendo de la aceptación (acrítica pensamos) de la ciudadanía a un conjunto de ideas y hábitos compartidos. Con ello se crean las instituciones educativas necesarias y suficientes que aseguren el orden social y eviten el vacío moral; a lo que Durkheim añadirá la función de adaptación como pre-requisito de la articulación social orgánica. ${ }^{4}$

Así, las primeras teorías sociológicas de la educación señalan la importancia de la educación como "subsistema social de aprendizaje de las normas y valores sociales en los que se fundamenta la sociedad" ${ }^{\prime \prime}$, pautando la transmisión de saberes y hábitos, del orden técnico y expresivo (discursivo) diría Foucault (1980). Tanto la primera sociología formal de la

4 Bonal, Xavier.(1998). Sociología de la educación. Una aproximación crítica a las corrientes contemporáneas, ed. Paidós, Col. Papeles de pedagogía, pp. 17, 18.

5 Ibidem., p. 18. 
educación, como el análisis genealógico foucaultiano, parecen enfocar la educación como una sociología de las instituciones escolares. La primera, en aras de contribuir a la armonización que condujera al orden y al progreso positivistas; la segunda, a desentrañar cómo la producción de saberes académicos contribuye a los procesos de normalización y control social.

Por otra parte, desde el siglo XIX nos dice Bonal:

Se trata, sin duda, de la importancia de la educación en el proceso de asignación y distribución de las posiciones sociales. En efecto, en la sociedad meritocrática capitalista la escuela adquiere una importancia fundamental como institución clave para la adquisición de estatus (p.19, 1988).

La herencia de Durkheim

En tiempos más recientes pero retomando algunas raíces de éstos clásicos de la Sociología, que no de la educación; se vino desarrollando en diversas latitudes del orbe, lo que podríamos denominar estudios sobre la educación. Por ejemplo, (De Azevedo, 1997) escribió un libro al que intituló Sociología de la educación y que fue publicado por vez primera en 1940. En la cuarta de forros de la edición 1997, el autor afirma que después de Durkheim "quien empezó la investigación sobre la sociología en su rama educativa.(...)..su obra se perdió entre las especulaciones pragmáticas que sus alumnos elaboraron confundiéndola con una sociología aplicada a la educación".

Se alude también en ese resumen a que últimamente otros autores han vuelto a la sociología de la educación estricta; pero señala que sus trabajos han quedado como meras monografías y apuntes que abordan aspectos sólo fragmentarios de la materia. Según la editorial (FCE, México), Azevedo se propuso además de abordar los procesos, instituciones y sistemas escolares", - la interdependencia que existe entre la estructura y la organización sociales, de una parte, y los procesos educativos de otra hacer el análisis de las teorías y doctrinas pedagógicas. Considerando también 
que, el libro es a la vez, un tratado, un libro de texto y una fuente de información. En efecto, es todo eso a través de un esfuerzo de sistematización y síntesis contribuyente al estudio científico (según su óptica), de los fenómenos pedagógicos y de sus relaciones con los demás fenómenos sociales. Decimos en nuestra opinión que es además una síntesis muy loable; por el hecho de que Azevedo —no dudamos aceptaría—, la cantidad de problemas propios de la pedagogía y su vinculación con otras dimensiones sociales, aunado a la cantidad de autores y propuestas que las discuten, proponen y critican entre sí - directa o indirectamente - , etc.; lo que hace de la problemática un estudio difícil para ser misión de un solo hombre, y para tan sólo una variante de la sociología.

Ahora bien, del otro lado del Atlántico, en los E. E. U. U., existió una revolución cultural con la obra y escuela de John Dewey la "sociología educativa". "La llamada "educational sociology", desarrollada desde departamentos de pedagogía de universidades norteamericanas, respondió a la auténtica revolución que experimentó el sistema educativo norteamericano de comienzos de siglo y a su función de preparación de masas de trabajadores para la creciente economía industrial". " Se dice que durante la primer época del siglo la "sociología educativa de Dewey surgió como alternativa a la orientación metafísica y esencialista dominante de la pedagogía (Jerez Mir, p.358, 1990). Con este autor, la educación es contemplada como un proceso activo y crítico fundamental para la cohesión social; acercándose con ello a la obra de Durkheim.

Sin embargo, y a manera de anécdota; con respecto a la discusión de los fines, mientras que la mayoría de los sociólogos asumimos como cierto el hecho de que la determinación social es preponderante a nivel macro con respecto al impacto en los fenómenos educativos, como Durkheim, o como Marx; Alain exponía por ejemplo, casi en la misma época que en

6 Ibidem., p. 23. 
éstos, es enorme el peso de la individualidad en la valoración por la educación. Esto demuestra que los discursos disciplinarios crean concepciones del mundo cerradas a otras fuentes proveedoras de conocimientos. Dicha parcialidad también ha sido ya señalada por los epistemólogos formados en la Dialéctica Crítica.

Otras visiones sociológicas de la educación.

Aunque no abundaremos en las varias sociologías de la educación (existen varios buenos estudios algunos citados, como para detenerme en ello); Pero si habremos de detenernos en los trabajos que dan cuenta de la evolución, crisis y estancamiento de los estudios e investigaciones educativas, para ir articulando la problemática.

Cabe hacer mención al asunto de los llamados paradigmas en el sentido de que, para nuestros objetivos sin embargo, por paradigmas entendemos a la manera de Khun, aquélla ciencia que se realiza con base en teorías probadas, las cuales sólo se aplican a un objeto que se pretende explicar, de ahí que en este trabajo no profundizaremos en ellos, toda vez que como se podrá ir viendo, la propuesta de cientificidad implica separarse de cualesquier predeterminación teórica.

Aunque como hemos dicho, no es el objetivo seguir profundizando en la sociología de la educación, cabe mencionar sin embargo, algunas de las corrientes contemporáneas más conocidas y los avatares de su evolución apoyados en estudios recientes, pues hay consenso en que es posterior a la segunda guerra mundial cuando se acrecienta la producción sociológica de la educación, apareciendo nuevos ámbitos, así como nuevos campos en la sociolgía de la educación. Entre ellos tenemos: el funcionalismo tecnoeconómico y la teoría del capital humano; el funcionalismo reformista: educación e igualdad de oportunidades; la teoría credencialista de Randall Collins; la teoría de la reproducción de Bordieu y Passeron; la sociología de las clases, códigos y el control de Bernstein; Educación y reproducción económica o del marxismo y sociología de la educación; Althusser: la 
educación como aparato ideológico del Estado; la teoría de las redes escolares de baudelot y Establet; la teoría de la correspondencia y su revisión.

Más recientemente, a partir de los años ochenta surgen nuevos paradigmas: el "paradigma interpretativo" al interior de la sociología de la educación; también el interaccionismo simbólico; la sociología del currículum; la teoría de la producción cultural y resistencia; la de la hegemonía y resistencia en la educación; las dimensiones de género y etnia; las formas de transmisión del sexismo en la escuela; y la escuela y la educación multicultural.

Actualmente la sociología de la educación ha constituido nuevos paradigmas: la sociedad de la información y la relación entre educación y empleo; la sociología de la política educativa; y, la de la educación y la atención a las diferencias (Bonal, 1998).

En este trabajo no se pretende tampoco, a partir de la búsqueda de los fundamentos de todas estas y otras perspectivas que seguramente abordan a la educación. Intentamos sólo exponer algunas de las muchas perspectivas que la abordan, para convencer de la necesidad de una proposición más abierta y por ello, más duradera y general para el abordaje de los distintos problemas de la educación. Por ello nos limitaremos a reseñar el escenario actual de la sociología de la educación.

\section{La investigación educativa (pedagógica e histórica)}

Es sabido que cualquier división de los tiempos históricos resulta más bien superficial con respecto de los efectos posibles de los procesos sociales; sin embargo, contribuyen a demarcar aproximadamente los pesos o los énfasis de dichos procesos. También es bien aceptado el hecho de que no existe fenómeno educativo alguno que no esté vinculado a las contingencias económicas y sociales. La investigación educativa no tendría porque escapar a ellas.

Partimos del supuesto de que en las diversas épocas históricas -ya sean demarcadas por acontecimientos sociales relevantes en la praxis hu- 
mana, y, o por efecto de los cambios en la historia de las ideas- Se puede decir que existen al menos dos grandes períodos que se distinguen bajo el concepto de modernidad, con base en la emergencia, en el siglo XIX, de la primacía de la razón humana sobre la perspectiva teológica como cosmovisión del mundo.

Con esto no se quiere decir que, la educación y el estudio sobre sus implicaciones sea totalmente diferente entre toda la producción mundial escrita al respecto hasta antes de la época de Kant, Hegel y Rousseau, pero si son significativos los cambios que la educación sufre a partir de estos tiempos, los cuales en la praxis humana se identifican con la primera revolución industrial, la que desarrolla un tipo de empresa y de burocracia de magnitudes nunca vistas en la historia de la humanidad. La producción manufacturera y la gestión se racionalizan para dar respuesta a sus necesidades, progresar y aportar el máximo beneficio.

“Este objetivo se logra mediante la aplicación de principios científicos de alto componente metrológico, cuya expresión más clara es posiblemente el taylorismo. Como lo recuerda A. León (p.78, 1980), J. M. Rice, quien fundó en 1903 la primera Society of Educacional research, apenas tres años después de la aparición de los Principles of Scientific Management de F. W. Taylor (1911), publicó su Scientífic Management in Education". (de Landsheere, p.11, 1996) ${ }^{7}$.

La educación se vuelve así un imperativo de las diversas clases sociales, lo que permite al fin fundar exigencias educacionales para todos. Lo que da paso a la exigencia de estudios e investigaciones sobre el currículo: ¿Qué enseñar?, ¿Cuánta enseñanza?, ¿Cómo ser eficaz? ¿Por qué se generan diferencias en los aprendizajes de los alumnos?, ¿Cómo evaluar resultados? Y ¿Cómo formar docentes en función de sus nuevas tareas?. Es decir desde

7 Partiendo del estudio de los obreros en el trabajo, Taylos, ingenieroestadounidense, elaboró un sistema que persigue esencialmente dos objetivos: aumentar el rendimiento y aumentar el desperdicio de tiempo. Sus principios son: La individualización del trabajo, fundada en el estudio psicológico del trabajador, la especialización en una sola función y la estandarización en función de criterios precisos. 
el punto de vista de una educación con base en la ciencia.

Y ya que hemos entrado en el terreno de los pedagogos; una aclaración conceptual sobre el asunto merece nuestra atención. Según Landsheere: A semejanza de una "psicología nueva" -nos dice de Landsheere (p.12-13, 1996) - ...se califica — a principios del XX — de "pedagogía nueva" a una amalgama de pedagogía experienciada con un alto componente empírico, de psicología pedagógica y de pedagogía experimental propiamente dicha...

La primera carece de rigor científico; la segunda confunde los campos de investigación. Sólo la tercera constituye el objeto particular de la presente historia. Pero esto no significa —lo que señalaremos en diversas ocasiones-, que las aportaciones de las otras dos modalidades sean desdeñables ni mucho menos. ${ }^{8}$

El enunciado de pedagogía experiencial deviene del siglo XIX, expresión que se forjó paralelamente a la noción de psicología experimental; y aunque, el término pedagogía limita el campo de investigación al niño -considerado sobre todo a éste en el medio escolar-, hoy la concepción de los tiempos de educación se han extendido. De otra parte, experimental connota un tipo preciso de proceder con la intencionalidad de modificación.

Según el autor antes citado, lo anterior es la causa de que la expresión investigación educativa (se sobreentiende que científica) se sustituye progresivamente por el término pedagogía experimental. No obstante, el término investigación educativa tiene evidentemente un significado más amplio, que los resultados empíricos - cuantitativos, producto de la pedagogía experimental. Como se verá, actualmente el enfoque cualitativo se reserva un mayor campo de acción a la investigación educativa. Sin duda, la historia,

8 De Landsheere, Gilbert (1996). La investigación educativa en el mundo, México, ed. F. C. E., pp. 13. 
la filosofía, la psicología y la sociología han incursionado con sus corpus teóricos en la realización de investigación educativa (IE).

Por lo anterior, es indispensable ir demarcando a nuestra vez, los linderos por los cuales ha de connotarse nuestro entendimiento en torno a la investigación educativa. La intencionalidad apunta a un enfoque de las diversas ciencias sociales pero articulados estos discursos en una lógica de aprehensión de lo real como totalidad; pero por ahora me centraré en decantar lo más precisamente, por el momento, las particularidades que implicamos en nuestra acepción de la (IE).

En principio, las preocupaciones arriba mencionadas de las investigaciones sobre el currículo: ¿Qué enseñar? ¿Cuánta enseñanza?, etcétera; las consideramos mayormente en su contenido social, como supeditada al contexto histórico en el cual se le estudia. Así mismo, la toma en cuenta de su historia (deviniente) y de su historicidad (coyuntura, actualidad, presente), el cual se funde con la perspectiva de futuro -el que involucra la praxis social-, es decir con el factor político, deben ser estudiados primeramente por las ciencias sociales. Y sin embargo, estos elementos no se contraponen con los estudios micro - pedagógicos; son su contorno explicativo y teorizante posibles.

Ahora bien, como se verá enseguida, estos planteamientos chocarán con las distinciones de Landsheere, quien por ejemplo acota:

Sin dejar de tomar en cuenta la importancia capital de las normas (sobre todo en el terreno cultural y de la filosofía), reflexión que está orientada a reunir, coordinar y a hacer coherentes no sólo los resultados de las ciencias particulares, sino también los de los demás órdenes del saber, a fin de alcanzar una concepción global del mundo, estructurada y unificadora" (Cardinet y Schmutz, p.15, 1975). 
No hemos considerado, en este trabajo, las investigaciones de tal índole. Estas se basan esencialmente en juicios de valor y escapan de ese modo al campo de la ciencia, que exige un acuerdo en función de los hechos (y no de las creencias) ${ }^{9}$.

Sin embargo, nuestro autor momentáneo, consciente de lo útil de la mayor acotación posible en la delimitación de los campos, lo asume volviendo aún más claro tanto el campo de los hechos que le interesa estudiar, así como la metodología implícita (de las ciencias exactas), que conlleva finalmente una definición posterior. "Bien mirado — dice el autor-, es la expresión investigación experimental educativa la que menos se presta a confusión" ${ }^{10}$

A manera de reflexión.

No obstante, la seguridad con la que el autor emite sus juicios sobre lo que debe ser y cómo se debe estudiar, no le autorizan a elevarlos a rango de ley o autoridad en la materia. Con un poco de modestia intelectual, debería decir que ese es un punto de vista entre otros posibles para el estudio de lo educativo y sólo de una parte. Si bien no puede negarse que ciertos experimentos pedagógicos pueden coadyuvar a la mejora de la educación, las ciencias encargadas de distinguir y explicar los contextos en que tales experimentos deban tener lugar; son sin duda alguna las ciencias sociales, incluyendo a la psicología social y al freudo marxismo. La psicopatología de la sociedad contemporánea señalada por Fromm; así como los problemas de malestar socio cultural denunciados por Freud y la unidimensionalidad puesta al descubierto por Marcuse, demuestran las grandes dimensiones de los problemas mundiales que ha generado la "evolución

\footnotetext{
9 El subrrayado es mío. La concepción de ciencia del autor es sin duda durkheimiana. Señala el énfasis que la ciencia debe partir de los hechos (sociales) punto de partida de las "Reglas del método sociológico" de su compatriota. Existe demasiada evidencia de las limitaciones de dicho paradigma, como para discutir sobre su efectividad en los estudios socioeducativos.

10 lbidem., p. 14.
} 
" de la sociedad moderna; a los cuales sin duda la investigación educativa ha de tomar en cuenta para intentar incidir en mejoras culturales; antes de innovar enmiendas a la educación que se transmite sin reflexión autocrítica de generación en generación.

De Landsheere, atrincherado en los fundamentos de la ciencia experimental (el viejo, viejo mapa de las ciencias), pretende que sólo debe llamarse investigación educativa a la que realiza experimentos y los prueba; oculta que el motor de qué se observa tiene un ineludible rasgo subjetivo (la creencia de que eso es lo que se debe de observar y experimentar. Dichos fundamentos implican una concepción estática y ahistórica del mundo, digna de la época del mecanicismo. El autor siente desprecio por los problemas filosóficos que circunadan al objeto educativo, así como por el pensamiento racionalista y crítico. Olvida que los cacareados "hechos de la ciencia" son producto de mediaciones sociales en donde los sujetos construyen su propia historia, por deleznable que esta sea en ocasiones. Su anacrónico método y su ideológica cosmovisión positivista del mundo, le impiden ver que las conciencias a educar son también productos sociales. Más aún, sus propios "principios" son resultado del alcance de sólo una mínima parte del conocimiento producido en una época específica.

Sin duda, la fragmentación del conocimiento inducida por el positivismo ha incidido negativamente en el especialismo, que se ha hecho famoso por su gran conocimiento sobre un "campo"; perdiendo de vista el andamiaje de la maquinaria de la totalidad social que, inercialmente arrolla a su paso todo intento de cambio a la maquinaria del establisment "cultural" actual. Pero a diferencia de la visión del marxismo diletante latinoamericano de los años setenta, aquí la burguesía no son sólo victimarios y la demás sociedad civil las victimas. Con el desastre ecológico mundial y la pauperización de la humanidad marginada, la ciencia positivista ha llevado a la totalidad mundial (incluyendo a las clases pudientes), a ver de cerca el fin del mundo, más allá de las predicciones bíblicas y, más acá de la realidad palpable. 
Supongo que se preguntarán ¿Por qué la crítica a la llamada "Investigación Educativa".? Las razones son varias, pero la más importante radica en que, es una visión que permea a la mayoría de los objetos de investigación en educación en la Región latinoamericana. Esta es fomentada por personas que asumen sin más el discurso experimental; el que teme y odia a cualquier discurso que no acepte sus premisas. Más allá de las posibles discusiones sobre la materia, están también los intereses grupales y los "clubes de cuates" en que se han constituido tales grupos, para constituirse a su vez en entes de presión al sistema. Al autopresentarse como los directrices de la llamada investigación educativa, se forman en primera fila para la consecusión de apoyos para sus "aportadoras" investigaciones — por lo menos en México—, según ellos científicas. Más aún, desde el centro geográfico en donde están al acecho del presupuesto, pretenden dictar línea de trabajo sobre los campos que deben ser prioridad en la IE. Los cuales no pasan de ser meros intentos de parches a la gran problemática educativa. Pronto estarán investigando cómo un niño analfabeta, marginado y en pobreza extrema puede aprender inglés y computación; para lo cual negociarán un puesto en el gobierno y, o pedirán recursos para realizar tan importante trabajo en el cual ya sería el colmo que creyeran.

O tal vez se lo creen por la excesiva falta de autocrítica. Se pasa por alto o se ignora, que existe una casi oculta división internacional del conocimiento: se recomienda a los países subdesarrollados institucionalizar un espíritu pragmático del conocimiento (el know how del uso tecnológico); mientras que los países líderes del mundo se apropian del derecho de erigirse como los productores del conocimiento. Ante esta grave problemática consideramos necesaria una: 


\section{Construcción de objetos socioeducativos con base epistemológica}

La propuesta incluye una concepción de temporalidad (el tiempo presente), un concepto de realidad (compleja y dinámica), y una lógica de descubrimiento, a partir de la articulación transdisciplinaria con base en la totalidad.

El presente como aquí lo concebimos, es un proceso dinámico que no cesa, donde fluye un haz de acontecimientos que determinan diversas especificidades. No nos dejemos engañar por la apariencia de la quietud del presente o por la errónea idea de que sobre él nada podemos. Es verdad que el presente se aparece como un haz de acontecimientos aislados sin una supuesta relación de necesidad existencial; pero el presente es contingente y sólo mediante un pensamiento abierto y crítico, es decir científico, se pueden desentrañar las relaciones necesarias entre ese mar convulso de acontecimientos.

La respuesta a esta representación del movimiento está en encontrar la relación entre lo que está dado y lo que está dándose (proceso). En dicha relación entre los momentos de la realidad, se encuentra la potencialidad que orienta la dirección de un campo de acción entre actores, pero esta no es manifiesta: Sin embargo es un contenido emergente. Lo que Hegel llamaría "el lugar de las transformaciones cualitativas". Lo que para Bloch sería "lo novum".

Esta dimensión está fuera de los alcances del paradigma disciplinario y del empirismo, toda vez que, "topos" (el lugar) es el objetivo empírico. Pensar la realidad como un topos, como un dato objetivo o con una perspectiva objetivista, diagnosticarlo y pronosticarlo a partir de la objetividad, es ya, estructuralmente, quedarse enraizado y sin avanzar, en el mismo lugar.

La totalidad. Recurso epistemológico para la articulación transdisciplinaria, significa en sentido simple, construir el conocimiento por articulación de niveles multidimensionales de lo real. Lo transdisciplinar se refiere en este planteamiento, al hecho de relevar "la cosa educativa o, socioeducativa", el objeto, la realidad educativa, por encima de cualquier discurso sustantivo disciplinario (incluyendo ramas concretas de la sociología 
de la educación). Transdisciplinario aquí es eso, abrir el pensamiento mas allá de los límites de estructuras teóricas preestablecidas, pero, sin desechar a priori, las posibilidades de captación de lo real por las categorías y conceptos de constructos teóricos ya construidos, se trata de actuar como dijera Zemelman, con una actitud crítica en su uso. Por ello proponemos esta forma de aprehensión de lo real(vía la totalidad); a diferencia de lo que sería cerrarse a las posibilidades de abstracción de dichos constructos teóricos, antes de ver sus potencialidades objetivas en el momento de la construcción del conocimiento socioeducativo.

Pensemos por analogía en la similitud que existe entre un individuo y una disciplina. Así como el individuo está constituido por diferentes referentes de lo real; una disciplina está también constituida por diferentes fundamentos epistemológicos: su concepción de la ciencia, su concepción ontológica, su concepto de realidad y su método o, su forma de aprehensión de lo real.

En su origen las disciplinas deambulan y se esfuerzan por constituirse con el estatuto de ciencia, para poder autopresentarse exitosamente. Es decir para lograr consenso de su legitimidad como disciplina científica, tal fue el caso de la Sociología en el Siglo XIX, y de otras ciencias sociales. A todas ellas sin embargo, se les ha cuestionado su estatuto de cientificidad desde la perspectiva "naturalista", y sin embargo se encuentran en todo el mundo occidental (por lo menos), aunque con diferentes matices $y, 0$ enfoques con diferentes fundamentos. No se trata aquí de rehacer la historia de las disciplinas científicas, se intenta problematizar el significado profundo de la totalidad como recurso epistemológico para el conocimiento, para tratar de dar validez a una propuesta: es decir, para proponer a la transdisciplina, como articulación disciplinaria vía la totalidad.

Vista así, la totalidad no es la suma de las partes, sino la inclusión de lo pertinente y la exclusión de lo irrelevante.

Lo dicho implica que la totalidad no son todos los hechos, sino que es una óptica epistemológica desde la que se delimitan campos de observación de la realidad, los cuales permiten reconocer la articulación en que los 
hechos asumen su significación específica. En este sentido se puede hablar de la totalidad como exigencia epistemológica del razonamiento analítico. (Zemelman, p.18, 1987)

La totalidad es un recurso para organizar el razonamiento analítico, no intenta formar un objeto 'total', que incluya el todo de todo, pues se centra en definir la base de la teorización, sin ser una teoría en sí misma; ni mucho menos pretende ser un objeto real. Es un mecanismo que puede organizar la apertura de nuestra mente hacia la realidad, sin ceñirse a pertenecer dentro de determinados corpus teóricos. Esto es así, porque se fundamenta en una concepción de lo real como articulación compleja de procesos exigiendo que cada uno de éstos sea analizado en términos de sus relaciones con otros -aunque no sean estos últimos objeto de análisis como tal—.

Por eso el concepto "todo" constituye un modo de recortar la realidad para construir el objeto de estudio que sin partir de exigencias a priori de "todas las propiedades o aspectos de una cosa", permita dar soluciones sobre "ciertas propiedades" o aspectos especiales de la cosa en cuestión...que le hacen aparecer como una estructura organizada...Por esto también consideramos que la totalidad representa una solución al asunto de la preeminencia del problema" por sobre cualquier ideal metodológico, esto es, sus mecanismos de adecuación a la cosa concreta. (Zemelman, p.18, 1987)

Es su capacidad de apertura hacia lo real lo que permite profundizar mayormente en la realidad con el objetivo de dar prioridad a la construcción del objeto. La totalidad así concebida deviene como un método que hace posible el recorte o construcción del objeto. Este último sin embargo nos plantea una importante pregunta.

¿Cuáles son los fundamentos de conocimiento implícitos que están en conexión con un concepto de totalidad de la forma que se plantea?

La necesidad de una apertura de la razón como actitud abierta a la especificidad de lo real, para el entendimiento de la interacción que existe 
entre la multiplicidad de objetos (incluyendo a sujetos sociales concretos) posibles en el mundo real. Estos y sus interacciones existentes son susceptibles de captación con base en los conceptos de especificidad, realidad dinámica y la perspectiva de totalidad como recurso epistemológico.

Para proceder tanto a la construcción del objeto (socioeducativo en este caso), así como a su teorización posible, Zemelman (1987) plantea la necesidad de tener un control de los condicionamientos de la razón que impiden la objetividad del conocimiento, imprimiendo sesgos en los análisis. Se intenta evitar las determinaciones a priori de sucesos con base en nuestro esquema referencial teórico, ideológico o de la experiencia, para dar paso a una visión que, concibiendo al mundo como un universo de la mayor complejidad, pueda descubrir lo específico concreto de las conexiones determinantes del hecho real.

Para ello se esbozan los mecanismos metodológicos para la construcción del objeto como reconstrucción articulada e histórica; aprehendiendo su especificidad con base en la articulación transdisciplinaria para el descubrimiento de las conexiones determinantes del objeto, con otras dimensiones de lo real.

Estos son en síntesis los elementos constitutitos de una propuesta, para la construcción (pero también para la teorización de objetos socioeducativos), que no se limiten a explicaciones simplistas con base en la prueba de una o dos hipótesis (método hipotético deductivo), pues partimos de que la realidad socioeducativa está más en espera de ser teorizada e incidida, que esperando simplemente ser explicada.

\section{Referencias:}

AUBENQUE, P. Aristóteles y el Liceo. En Historie de la philosophie, Enciclopédie de la Pléiade, vol. I, París, Gallimard, 1969.

CHARTIER, Emile. Propos sur l'èducation, PUF, colección "Quadrige", 1986.

BELLO, Andrés. Obras Completas de Andrés Bello. Ed. "La Casa de Bello", Tomo XVIII, Temas educacionales, 1919. 
B, Bettelheim, M, Y Janowitz. Cambio social y prejuicio, México: Fondo de Cultura Económica, 1964.

BINET, Alfred. Les idées modernes sur les enfants. París: Flammarion. Reeditado en este año con prólogo de Jean Piaget, 1973.

BLONSKY, P. P. XVIII Congreso Internacional de Psicología. Aprendizaje y Desarrollo Intelectual, Moscú, 1966.

BONAL, Xavier. Sociología de la educación. Una aproximación crítica a las corrientes contemporáneas, Ed. PAIDÓS. Papeles de Pedagogía, No. 37, 1998.

BUBER, Martin. On intersubjetivity and cultural creativity. Chicago: University Press, 1992.

CLAPAREDE, Edouard. Quelques mots sur le Collège de Genève, Stapelmor, 1982.

JOBERT, J. C. Don Andrés Bello, orientador de la enseñanza de la época. En Doctrina y praxis de los educadores representativos chilenos, Santiago: Ed. Andrés Bello, 1970.

CONDORCET. Reflexions et notes sur I'éducation, Bibliopolis, Nápoles, 1983.

De Landsheere (1996). La investigación educativa en el mundo, Ed. Fondo de Cultura Económica.

DURKHEIM, Emile. Education et sociología, París, PUF, 1992.

DURKHEIM, Emile. L'évolution pédagogique en France, París PUF, 1992.

FREINET, Célestin. L'école moderne francaise. Montmorillon, Editions Rossignol, 1957.

FREIRE, Paulo. La educación: práctica de la libertad. Rio de Janeiro, Paz e Terra, 1965.

FREIRE, Paulo. Educación para el cambio social. Buenos Aires, Tierra Nueva, 1974.

FREUD, Sigmund. Presentación autobiográfica, Buenos Aires, Amorrortu, 1976.

GRAMSCI, A. La alternativa pedagógica. Barcelona, Fontamara., 1981.

GRAMSCI, A. Los intelectuales y la organización de la cultura. Buenos Aires, Nueva Visión, 1984.

HEGEL, G. Fenomenología del Espíritu. México, F. C. E, 1966.

HEGEL, G. Principios de la filosofía del derecho. Buenos Aires, Sudamericana, 1975.

ILLICH, I. En América latina ipara qué sirve la escuela? Buenos Aires, Ediciones Búsqueda, 1973.

ILLICH, I. La sociedad desescolarizada., Barcelona. Barral Editores, 1974. 
ELHRLICH, L. H.; WISSER, R. (Comps.). Karl Jaspers Today: Philosophy at the Thershold of the Future. Nueva York, NY, (Current Continental Research Series, 10), 1988.

KANT, Immanuel. La filosofía de la historia. México, F. C. E, 1978.

MASSÉ, Carlos. La complejidad de las ciencias sociales en la sociedad de la información y la economía del conocimiento. Trastocamiento objetual y desarrollo informacional, ed. El Colegio Mexiquense, A.C, 2005.

MASSÉ, Carlos y PEDROZA, René. La complejidad en las ciencias. Método, institucionalización y enseñanza, ed. El Colegio Mexiquense, A. C, 2002.

ZEMELMAN, M. Hugo. Uso crítico de la teoría, Universidad de las Nacions Unidas-El Colegio de México, 1987.

\section{Resumen}

El trabajo se centra en cuestionar, por qué en el país (independientemente de que el problema, seguro impregna otras latitudes latinoamericanas) la Ilamada "Investigación Educativa" (IE) adoptó acríticamente a la llamada teoría educativa y, o a la teoría pedagógica clássica. Asumimos la posición de que una pretendida perspectiva de base "experimental" no puede autoproclamarse poseedora del herramental de conocimiento para la investigación educativa y, o declarar que ese es El Paradigma correcto. Para ello hacemos un breve ejercicio de problematización de algunas de las múltiples dimensiones que abarcan, o abarcar pordrían, algo así como El Campo de La Educación. Posteriormente, pasamos a esbozar una propuesta que releva a la construcción de conocimiento de dicho campo, en los puntos de articulación que quieren ser conocidos, pasando por el cuestionamiento y el control del elemento explicativo de las teorías educativas y sociales en general, para subrayar la necesidad de un uso crítico de estas, así como un uso crítico de los factores Ideológico, el "experiencial", como factores predeterminantes muchas veces a priori.

Palabras-clave: Teoria, Epistemología, Investigación Educativa, Conocimiento, Ideología, Totalidad, Articulación, Transdisciplinariedad, etc.

Recebido: 06/03/07 


\section{The construction of epistemologically based socio-educational objects}

\section{Carlos E. Massé Narváez}

The article questions why, in Mexico (independent of the fact that this problem also occurs in other Latin American latitudes), the so-called "educational research" (ER) has uncritically adopted the educational theory and/or the classical pedagogical theory. This work maintains that the proposed perspective of an "experimental" basis cannot presume to possess the knowledge tools for educational research, or state that this paradigm is the right one. For this reason, the text presents a brief exercise of problematization of some of the multiple dimensions that include, or could include, something like the field of education. Subsequently, the paper outlines a proposal that deals with the construction of knowledge in this field at its key points of articulation, which include the questioning and control of the explanatory element of the general educational and social theories, to emphasize the need for a critical use of these theories, and a critical use of the ideological factors, the "experiential," as predetermining factors, often a priori.

Keywords: theory, epistemology, educational research, knowledge, ideology, transdisciplinarity. 